\title{
Ground-state energy of a classical artificial molecule
}

\author{
L. Cândido ${ }^{1, a}$, T.L. Fonseca ${ }^{1}$, J.N. Teixeira Rabelo ${ }^{1}$, and G.-Q. Hai ${ }^{2}$ \\ 1 Instituto de Física, Universidade Federal de Goiás Campus Samambaia, 74001-970 Goiânia, GO, Brazil \\ 2 Instituto de Física de São Carlos, Universidade de São Paulo, 13560-970 São Carlos, SP, Brazil
}

Received 17 April 2008 / Received in final form 9 June 2008

Published online 16 July 2008 - (c) EDP Sciences, Società Italiana di Fisica, Springer-Verlag 2008

\begin{abstract}
We study the ground-state energy of a classical artificial molecule formed by two-dimensional clusters (artificial atoms) of $N / 2$ charged particles separated by a distance $d$. For the small molecules of $N=2$ and 4, we obtain analytical expressions for this energy. For the larger ones, we calculate the ground-state energy using molecular dynamics simulation for $N$ up to 128. From our numerical results, we are able to find out a function to approximate the ground-state energy of the molecules covering the range from atoms to molecules for any inter-atom distance $d$ and for particle number from $N=8$ to 128 within a difference less than one percent from the MD data.
\end{abstract}

PACS. 36.40.Wa Charged clusters - 02.70.Ns Molecular dynamics and particle methods - 68.65.Hb Quantum dots - 71.10.-w Theories and models of many-electron systems

\section{Introduction}

Models of clusters constituted of a small number of spatially confined interacting charged particles obeying classical mechanics have been used to study a wide variety of systems in different fields ranging from astrophysics to condensed matter physics. Such models can for instance represent colloidal particles in a circular trap [1], ions trapped by laser beams [2], charged dust particles in plasma traps [3], spherical Coulomb crystal of ultra-cold ion plasmas [4], vortices in superfluids [5], electron dimples on the surface of liquid helium [6], as well as electrons in quantum dot [7]. Spherical Wigner crystal in the interior of white dwarf stars is also expected to be described by such a model. Experimentally, the creation of such confined systems is currently a daily reality. One of the great success attained is mainly the electron confinement in semiconductor structures due to the manipulation of the semiconductor materials through the development of new technologies of their preparation and growth [8-10]. Such structures are the so-called quantum dots or "artificial atoms" which have interesting optical and transport properties and are of interest in single-electron devices. A pair of interacting quantum dots can form a so-called "artificial molecule". The two quantum dots can couple to each other laterally or vertically according to their relative geometrical position forming different artificial molecules. Many interesting properties have been explored in these systems. Theoretically, there is no a simple approach to deal with such a system because both the many-body effects and

\footnotetext{
a e-mail: ladir@fis.ufg.br
}

the behavior of each individual electron play important roles in the determination of more realistic properties. As a consequence, a common approach treating the charges as a continuum distribution is not appropriate. Therefore, in order to understand the role of Coulomb interaction effects in such a system, it was treated classically in which the particles are taken point-like but no approximation was made on the Coulomb correlation [11]. Numerical methods have been applied such as classical Monte Carlo [11] (MC) and molecular dynamics [12] (MD). The results obtained from these methods have shown that the charge particles in a classical atom arrange into shell structures similar to the Mendeleev's table [11,12] and so as resembling the charge distribution by Thomson in his model of atom [13]. In addition, it was shown that a system of two vertically or laterally coupled classical atoms or a classical artificial molecule displays several structural transitions of first and second orders $[14,15]$. Furthermore, in a study on the structure and Coulomb correlations in such classical artificial atoms [16], the MC results are compared with those of the hydrodynamics approach. In the hydrodynamics approach, the electron density is taken as a fluid, i.e., there is no charge quanta and, consequently, no correlation effects. It clearly shows the importance of the Coulomb correlation effects in such classical systems.

The present classical results are valid to study the quantum systems in the high magnetic field regime where the kinetic energy of the electrons is quenched. As a matter of fact, the equilibrium configurations of a classical artificial atom have been served to construct the trial wavefunctions of two-dimensional $N$-electron systems in high magnetic fields with long-range Coulomb correlations [17]. 
In this case, the unrestrict Hartree-Fock orbitals can be approximated by analytic Guassian functions centered at different positions determined by the classical calculations.

In this work, we study the ground-state energy of the classical artificial molecules both analytically and numerically. We employ the MD method to calculate the groundstate energy of a classical artificial molecule at zero temperature. Our calculation is in excellent agreement with available results in previous work $[11,12,14]$. We focus our attention on a symmetric molecule which is composed of two vertically coupled identical classical atoms. For small molecules, we obtain analytical expressions for the exact ground-state energy. For larger ones, from our MD data, we are able to find out a simple function with a few fitting parameters to give the ground-state energy of such a classical molecule including particle-particle Coulomb interaction. Our results are valid for the molecules of a few to more than one hundred charged particles within at most one or two percent difference only compared with the MD data. Such a function is desirable for studying the properties of a molecule because there is no a simple approach to deal with this kind of system in which both the many-body effects and the behavior of each individual particles play important roles. For instance, in order to obtain a fitting function for the correlation energy and correlation potential in a real atom or molecule, several density-functional formulas with some fitting parameters were proposed and widely used [18]. For an artificial molecule, however, there is no such a proposed fitting function in the literature so far to approximate its ground-state energy. Our simple formula for the ground-state energy is helpful to understand the physics of the inter-atomic Coulomb interaction which is the main responsible for driving the structural transitions in the atomic configurations, and consequently stabilizing the molecular structure. The results presented here complement the previous work [14] on such an artificial molecule.

\section{The model system and numerical approach}

The molecule is constituted of two classical artificial atoms coupled vertically in the $z$ direction and separated by a distance $d$. Each artificial atom is formed by a cluster of point-like charged particles moving in the $x y$ plane and kept together by a parabolic external potential. The Hamiltonian of the system is given by

$$
\begin{gathered}
H=\sum_{i=1}^{N_{a}} \frac{1}{2} \dot{r}_{i}^{2}+\sum_{i=1}^{N_{a}} r_{i}^{2}+\sum_{i=1}^{N_{b}} \frac{1}{2} \dot{r}_{i}^{2}+\sum_{i=1}^{N_{b}} r_{i}^{2} \\
+\sum_{i<j}^{N_{a}} \frac{1}{\left|\mathbf{r}_{i}^{a}-\mathbf{r}_{j}^{a}\right|}+\sum_{i<j}^{N_{b}} \frac{1}{\left|\mathbf{r}_{i}^{b}-\mathbf{r}_{j}^{b}\right|}+\sum_{i<j}^{N_{a, b}} \frac{1}{\left[\left|\mathbf{r}_{i}^{a}-\mathbf{r}_{j}^{b}\right|^{2}+d^{2}\right]^{\frac{1}{2}}}
\end{gathered}
$$

where $\mathbf{r}_{i}=\left(x_{i}, y_{i}\right), N_{a}$ and $N_{b}$ are the numbers of particles in the atoms $a$ and $b$, respectively. The above
Hamiltonian is written in reduced units so as to make it useful for different real systems at different scales. The units for length, energy, time, and temperature are defined by $\sigma=\left(e^{2} / \epsilon\right)^{1 / 3} \nu^{-1 / 3}, E_{0}=\left(e^{2} / \epsilon\right)^{2 / 3} \nu^{1 / 3}, \tau=$ $\left(m \epsilon \sigma^{3} / e^{2}\right)^{1 / 2}$ and $T_{0}=\left(e^{2} / \epsilon\right)^{2 / 3} \nu^{1 / 3} k_{B}^{-1}$, respectively, where $\nu=m \omega_{0}^{2} / 2, m$ is the mass of the particle of electric charge $e, \epsilon$ the dielectric constant of the medium, and $\omega_{0}$ is the confinement frequency of the clusters or atoms. Thus, the Hamiltonian is a function of two independent parameters only, the particle numbers and the distance $d$ between the atoms. As an example, for electrons in a GaAs heterostructure with a confinement energy of $h \omega_{0}=1 \mathrm{meV}$, the typical values for such reduced units are $\sigma=630 \AA$, $E_{0}=1.7 \mathrm{meV}, T_{0}=20 \mathrm{~K}$ [4]. The first and the second (the third and the fourth) terms are the kinetic energy and the confinement potential, respectively, of the particles in the atom $a(b)$. The fifth and sixth terms are the Coulomb repulsion between charged particles in the same atom and the last term is the inter-atom interaction. In this work, we study the symmetrical artificial molecules, i.e. $N_{a}=N_{b}$.

In order to obtain the ground-state energy of the system, we have used the MD simulations with a fourth order Predictor-Corrector algorithm for the integration of the Newton's equation of motion with a time step varying from $\Delta \tau=2.5 \times 10^{-3}$ to $5.0 \times 10^{-4}$. The MD procedure for the calculation of the ground-state energy at zero temperature was performed in three steps. Firstly, the system initial conditions are setup by placing the particles randomly in a circle with velocities according to the Boltzmann distribution function at high temperatures. Next, after the system reaches the thermodynamic equilibrium at a high temperature, the velocities of the particles are systematically decreased by a factor of $5 \%$, at each 50 times steps during several thousand time step runs of integration of the equations of motion, until the temperature reaches $T \sim 10^{-10} T_{0}$ which is practically zero temperature. Finally, after the thermalization, several different initial conditions are used to make sure that the ground state energy has been reached. Subsequently, the physical quantities are obtained by averaging over 100 blocks of 10000 time steps.

\section{Numerical results and theoretical analysis}

We have performed the MD simulations for two vertically coupled atoms with $N_{a}=N_{b}=1,2,4,8,16,32$ and 64 particles as a function of the distance $d$ between the atoms. The total number of charged particles in the artificial molecule is $N=2 N_{a}$. Table 1 summarizes our results for the molecule ground-state energy per particle, $\varepsilon^{(N)}=E^{(N)} / N$ for different inter-atom distances. At $d=0$, we recover the energy of an atom of $N$ particles $\varepsilon_{0}^{(N)}$. The energy value decreases with increasing of the inter-atom distance and tends to the energy value of a single atom of $N / 2$ particles (i.e., $\varepsilon_{0}^{(N / 2)}$ ) in the limit of large distances. The MD results for the total energy in the 
Table 1. The molecule ground-state energy per particle from the MD simulation and from equation (8) for several values of $N$ as a function of $d$. Also shown is the exact result for $N=4$ obtained from equation (5).

\begin{tabular}{ccccccccccccc}
\hline$d$ & \multicolumn{2}{c}{$N=4$} & \multicolumn{2}{c}{$N=8$} & \multicolumn{2}{c}{$N=16$} & \multicolumn{2}{c}{$N=32$} & \multicolumn{2}{c}{$N=64$} & \multicolumn{2}{c}{$N=128$} \\
& Exact & Eq. (8) & MD & Eq. (8) & MD & Eq. (8) & MD & Eq. (8) & MD & Eq. (8) & MD & Eq. (8) \\
\hline .0 & 1.8354 & 1.8354 & 3.6689 & 3.6689 & 6.6499 & 6.6499 & 11.4466 & 11.4466 & 19.1472 & 19.1472 & 31.4711 & 31.4711 \\
.25 & 1.8126 & 1.8167 & 3.6476 & 3.6592 & 6.6074 & 6.5856 & 11.3774 & 11.3445 & 19.0430 & 18.9614 & 31.3176 & 31.2164 \\
.50 & 1.7471 & 1.7418 & 3.5807 & 3.5731 & 6.4503 & 6.4551 & 11.1588 & 11.1760 & 18.7404 & 18.7159 & 30.8853 & 30.9021 \\
.75 & 1.6495 & 1.6559 & 3.3899 & 3.3956 & 6.2379 & 6.2474 & 10.8576 & 10.8960 & 18.3275 & 18.3759 & 30.3479 & 30.4815 \\
1.0 & 1.5391 & 1.5388 & 3.2114 & 3.2038 & 5.9983 & 5.9917 & 10.5414 & 10.5222 & 17.9096 & 17.9211 & 29.8014 & 29.8919 \\
2.0 & 1.2204 & 1.2248 & 2.7152 & 2.7136 & 5.2759 & 5.2719 & 9.5319 & 9.4945 & 16.5147 & 16.4045 & 27.9087 & 27.6313 \\
3.0 & 1.0743 & 1.0754 & 2.4620 & 2.4618 & 4.8591 & 4.8603 & 8.8726 & 8.8632 & 15.5158 & 15.3940 & 26.4491 & 26.3087 \\
4.0 & 0.9962 & 0.9966 & 2.3177 & 2.3178 & 4.6028 & 4.5993 & 8.4344 & 8.4238 & 14.7995 & 14.7593 & 25.3289 & 25.2146 \\
5.0 & 0.9480 & 0.9482 & 2.2261 & 2.2262 & 4.4334 & 4.4320 & 8.1307 & 8.1273 & 14.2759 & 14.2625 & 24.4647 & 24.4272 \\
6.0 & 0.9155 & 0.9156 & 2.1633 & 2.1634 & 4.3145 & 4.3138 & 7.9121 & 7.9099 & 13.8837 & 13.8792 & 23.7897 & 23.7811 \\
7.0 & 0.8921 & 0.8922 & 2.1177 & 2.1178 & 4.2268 & 4.2265 & 7.7458 & 7.7456 & 13.5804 & 13.5799 & 23.2533 & 23.2557 \\
8.0 & 0.8745 & 0.8746 & 2.0831 & 2.0832 & 4.1597 & 4.1595 & 7.6178 & 7.6179 & 13.3414 & 13.3422 & 22.8201 & 22.8264 \\
9.0 & 0.8608 & 0.8608 & 2.0560 & 2.0561 & 4.1069 & 4.1067 & 7.5171 & 7.5161 & 13.1487 & 13.1499 & 22.4649 & 22.4719 \\
10. & 0.8498 & 0.8498 & 2.0342 & 2.0343 & 4.0641 & 4.0641 & 7.4342 & 7.4332 & 12.9911 & 12.9917 & 22.1694 & 22.1759 \\
. &. &. &. &. &. &. &. &. &. &. &. \\
. &. &. &. &. &. &. &. &. &. &. &. &. \\
. &. &. &. &. &. &. &. &. &. &. &. \\
$\infty$ & 0.7500 & 0.7500 & 1.8355 & 1.8355 & 3.6689 & 3.6689 & 6.6499 & 6.6499 & 11.4466 & 11.4466 & 19.1472 & 19.1472 \\
\hline
\end{tabular}

limits of $d=0$ and $d \rightarrow \infty$ are in very good agreement with previous MC simulation results [4].

For a small artificial molecule, an analytical solution can be obtained for the ground-state energy. In particular, for $N=2$ and 4 , we can easily determine the configurations of the charged particles in the molecule using the equilibrium condition of the inter-particle Coulomb repulsion and the harmonic confinement at zero temperature. For $N=2$, if one particle in the molecule (one particle in each atom) is localized at $(R, 0,0)$, the other must be at $(-R, 0, d)$. The distance between them is $\sqrt{d^{2}+4 R^{2}}$. Then, the relation between $R$ and $d$ is given by

$$
R=\left\{\begin{aligned}
\frac{1}{2} \sqrt{1-d^{2}}, & \text { for } 1 \geq d \geq 0 \\
0, & \text { for } d>1
\end{aligned}\right.
$$

This leads to an analytic expression for the energy as a function of $d$,

$$
\varepsilon^{(2)}(d)=\left\{\begin{aligned}
\frac{1}{4}\left(3-d^{2}\right), & \text { for } 1 \geq d \geq 0 \\
\frac{1}{2 d}, & \text { for } d>1
\end{aligned}\right.
$$

At $d=0$, we obtain $\varepsilon^{(2)}(0)=\varepsilon_{0}^{(2)}=0.75$ which is the energy of a single atom of two particles.

For the molecule of 4 particles, if two particles are localized at $( \pm R, 0,0)$ in one atom, the other two particles should be at $(0, \pm R, d)$ in the other. We find that the relation between $R$ and $d$ is determined by the following equation,

$$
\left(\left(R^{3}-\frac{1}{8}\right)^{-\frac{2}{3}}-2\right) R^{2}-d^{2}=0, \text { for } \infty>d \geq 0
$$

At $d=0$ and $d \rightarrow \infty$, we obtain $R=\left[\frac{1}{2}\left(\frac{1}{4}+\frac{1}{\sqrt{2}}\right)\right]^{\frac{1}{3}}=$ 0.782186 and $R=\frac{1}{2}$, respectively. The ground state en- ergy of the molecule is given by

$$
\varepsilon^{(4)}(d)=R^{2}+\frac{1}{4 R}+\frac{1}{\sqrt{d^{2}+2 R^{2}}} .
$$

For $d=0(R=0.782186)$, we obtain $\varepsilon^{(4)}(0)=\varepsilon_{0}^{(4)}=$ 1.835445 which is the ground-state energy of a single atom of 4 particles. For $d \gg R$, this energy can be approximated by

$$
\varepsilon^{(4)}(d) \simeq \frac{3}{4}+\frac{1}{\sqrt{d^{2}+0.5}} .
$$

For $d \rightarrow \infty(R=0.5)$, we recover again from equation (5) the ground-state energy of a single atom of 2 particles $\varepsilon^{(4)}(\infty)=\varepsilon_{0}^{(2)}=0.75$. Our MD data for $N=2$ and 4 are in good agreement with the above analytic results.

For a larger molecule, it becomes difficult to obtain an analytical expression for the energy of the system as a function of the inter-atom distance. In order to obtain an expression for the molecule energy dependence on $d$ and $N$, we have to fit the MD results. Such a fitting function is helpful to understand the structural properties of a molecule and the effects of inter-particle Coulomb interaction between particles in different atoms. We notice that the number of inter-particle interaction pairs between the different atoms is $(N / 2)^{2}$. When the distance between the atoms is large, the energy can be approximated by the following expression,

$$
\varepsilon^{(N)} \rightarrow \frac{N}{4} \frac{1}{\sqrt{d^{2}+2 r_{0}^{2}}}+\varepsilon_{0}^{(N / 2)} .
$$

We fit first the MD data for $d \geq 5$ by the above function with $r_{0}$ as a fitting parameter. The obtained values for $r_{0}$ are given in Table 2. The energies obtained from equation (7) for systems of $N=8$ and 64 particles are represented by the dashed curves in Figure 1. One can 
Table 2. Parameters for the molecule energy function equation (8) determined by a non-linear least-square fitting.

\begin{tabular}{cccc}
\hline & $r_{0}$ & $\alpha$ & $\beta$ \\
\hline$N=4$ & 0.5005 & 0.0275 & 0.2397 \\
$N=8$ & 0.7848 & 0.0295 & 0.1376 \\
$N=16$ & 1.0918 & 0.1591 & -0.1234 \\
$N=32$ & 1.4484 & 0.4012 & -0.2923 \\
$N=64$ & 1.8797 & 1.1959 & -0.6368 \\
$N=128$ & 2.3907 & 2.7163 & -0.9148 \\
\hline
\end{tabular}

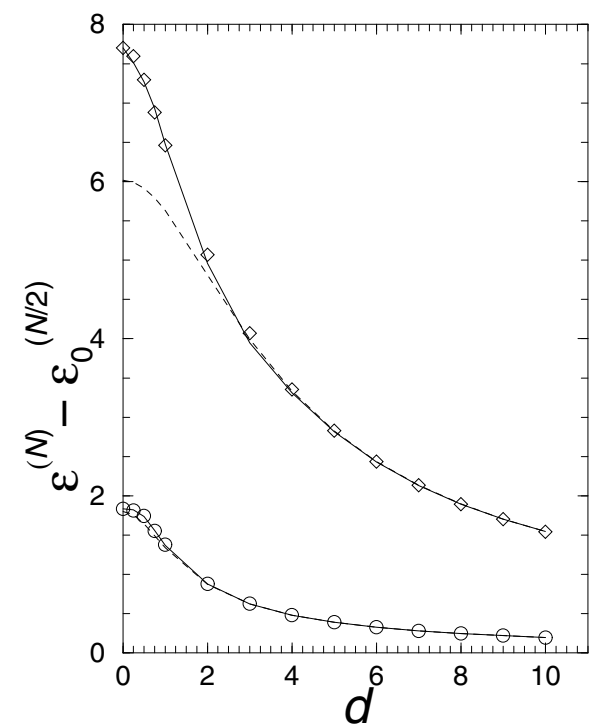

Fig. 1. The molecule ground-state energy per particle as function of the distance $d$ between the two atoms for the total charge particle number $N=8$ (open circles) and $N=64$ (diamonds). The dashed curves are given by equation (7) and the solid curves are fitting function given by equation (8).

observe that equation (7) is good even for $d \leq 5$ when $N$ is small.

Next, we fit the MD data $\varepsilon_{M D}^{(N)}$ for $0<d<\infty$ by the following function

$\varepsilon^{(N)}(d)=\frac{N}{4 \sqrt{d^{2}+2 r_{0}^{2}}}+\alpha \frac{\tanh \left[\alpha^{-1}\left(\lambda d^{3}+\beta d^{4}\right)\right]}{d^{3}}+\varepsilon_{0}^{(N / 2)}$,

where the particle-number dependent parameters $\alpha$ and $\beta$ are determined by a nonlinear least square fit to the MD data for different $N$. The parameter $\lambda$ is chosen as $\lambda=\varepsilon_{0}^{(N)}-\varepsilon_{0}^{(N / 2)}-N \sqrt{2} / 8 r_{0}$ to guarantee $\varepsilon^{(N)}(0)=\varepsilon_{0}^{(N)}$. The second term in the right hand side of equation (8) is a correction to the total energy at small $d$. It decreases as $\sim d^{-3}$ with increasing $d$ which is desirable for large interatom distances. The obtained values of the parameters $\alpha$ and $\beta$ that give the best fit results are listed in Table 2 . Equation (8) is able to fit all the MD data from $d=0$ to $\infty$ very well as is shown in Table 1 . For small $N$, the largest difference between equation (8) and the MD data appears around $d \approx 1$ which is a fraction of $1 \%$. For $N=64$ and 128 , this difference is a little larger being close to $1 \%$ at $d=1 \sim 3$. But it decreases rapidly with increasing $d$.
The parameters $r_{0}, \alpha$ and $\beta$ are $N$ dependent. Therefore, the success of equation (8) still depends on which formulas we can find out for these parameters as a simple function of the particle number $N$. In order to extract such a dependence, they are shown as open circles in Figure 2 as a function of $N$. For $r_{0}$, we tried different expressions for its dependence on $N$ and found that it can be describe very well as a linear function of $N^{\frac{1}{4}}$, given by

$$
r_{0}=\sigma N^{\frac{1}{4}}+\eta
$$

where $\sigma=0.9638$ and $\eta=-0.8460$. This function is depicted in Figure 2a as a solid line. As to the parameters $\alpha$ and $\beta$, their values affect the energy of the system at small $d$ only. A slight shift of their values from those given in Table 2 (the open circles in Figs. $2 \mathrm{a}$ and $2 \mathrm{~b}$ ) leads to a change on the energy in equation (8). In the figures, we indicate the range of the changes on both $\alpha$ and $\beta$ keeping the energy difference between equation (8) and the MD data less than $| \pm 1 \%|$ for $\infty>d \geq 0$. It means that any values of $\alpha$ and $\beta$ in the indicated ranges in the figures yield an energy according to equation (8) whose difference from the MD data is less than $1 \%$ for any $d$. In fact, the largest difference (close to $1 \%$ ) occurs at small $d$ only. Within a small global error in the total energy, we find surprisedly that these two parameters $\alpha$ and $\beta$ can be described very well by the following simple functions of the particle number $N$,

$$
\alpha=(N / 64)^{\frac{3}{2}}, \text { and } \beta=\frac{3}{4}(1-\log N),
$$

respectively. In Figures $2 \mathrm{~b}$ and $2 \mathrm{c}$, they are shown as the solid lines. The above functions for $\alpha$ and $\beta$ lead to a total energy of the artificial molecule within $1 \%$ difference from the MD simulation results for $N \geq 8$. For $N=4$, this difference is about $2 \%$ at $d<1$, but it is smaller than $1 \%$ for $d>1$.

In summary, from equation (8) with $r_{0}$ given by equation (9) and $\alpha$ and $\beta$ given by equation (10), we can obtain the ground-state energy of the artificial molecules within a global error less than $1 \%$ for $N=8$ to 128 , and less than $2 \%$ for $N=4$. We also find that, for $N<32$, the second derivative of the ground-state energy given by equation (8) with respect to the inter-atom distance $d$ shows a local maximum at small $d$ indicating the structural transitions of first order which were found in reference [14].

We now turn our attention to equation (8) in the limit cases of inter-atom separation. We take the limit of this equation for $d \rightarrow \infty$ :

$$
\begin{aligned}
\lim _{d \rightarrow \infty} \varepsilon^{(N)}(d)= & \varepsilon_{0}^{(N / 2)}+\frac{N}{4} d^{-1}-\left(\frac{N}{4} r_{0}^{2}-\operatorname{sign}(\beta) \alpha\right) d^{-3} \\
& +\frac{3 N}{8} r_{0}^{4} d^{-5}+O\left(d^{-7}\right)
\end{aligned}
$$

In this limit, we would expect a predominant Coulomb interaction. Within the $d^{-1}$ term, the molecule can be considered as two point charges separated by a distance $d$ with $N / 2$ charged particles at each point. The total energy due to direct Coulomb repulsion of the two point charges is 

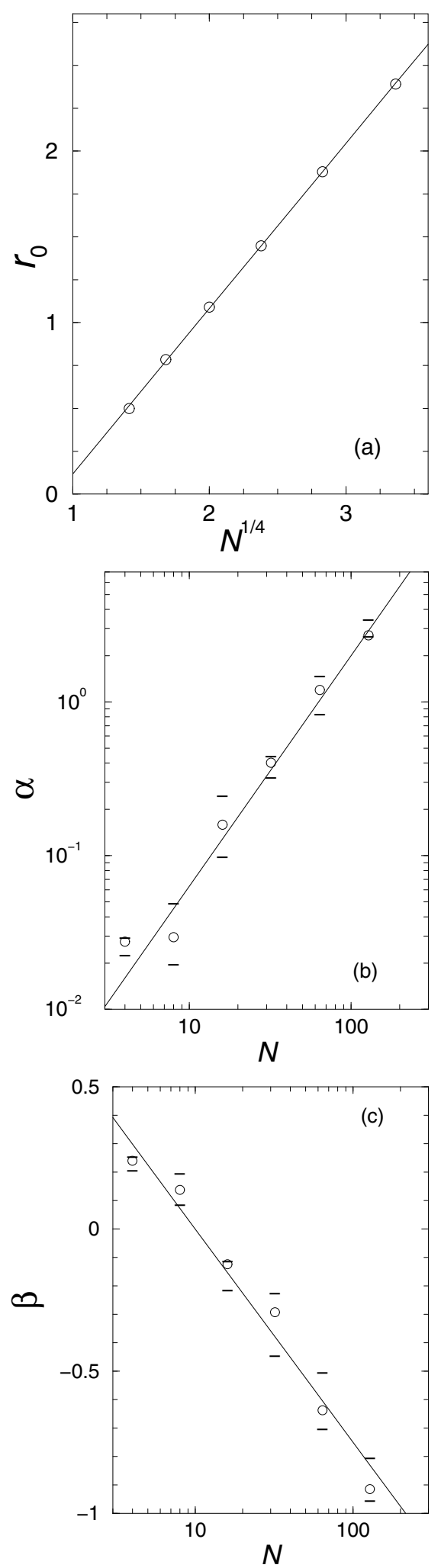

Fig. 2. The parameters (a) $r_{0}$, (b) $\alpha$, and (c) $\beta$ introduced in equation (8) against the total particle number $N$ in the artificial molecule. The open circles are from Table 2 . The solid lines are the functions given by equations (9) and (10). The two horizontal bars for a fixed $N$ indicate the range on both $\alpha$ and $\beta$ keeping the energy difference between equation (8) and the MD data less than $| \pm 1| \%$.
$(N / 2)^{2} d^{-1}$ which is just the $d^{-1}$ term in the above expansion (notice that the energy given in Eq. (11) is normalized by $N)$. The term $d^{-3}$ is a dipole-like potential between the two clusters. The contribution of this term to the total energy can be written as $\left[\left(\frac{N}{4}\right)^{2}\left(2 r_{0}\right)^{2}-\operatorname{sign}(\beta) N \alpha\right] d^{-3}$ which is basically a dipole-dipole interaction energy of two dipoles of charge $N / 4$ and dipole moment $(N / 4)\left(2 r_{0}\right)[19]$. The parameters $\alpha$ and $\beta$ contribute a correction to the dipole-dipole interaction which is originate from the intercluster or inter-atom correlation effects. This correction can be positive or negative depending on the sign of $\beta$ $(\beta>0$ for $N<10)$. But it is a small correction so that the dipole-dipole interaction is always repulsive in this case. The $d^{-5}$ term is a quadrupole contribution depending on $r_{0}$ only.

In the $d \rightarrow 0$ limit, we obtain

$$
\lim _{d \rightarrow 0} \varepsilon^{(N)}(d)=\varepsilon_{0}^{(N)}+\beta d-\frac{\sqrt{2} N}{32} \frac{d^{2}}{r_{0}^{3}}+O\left(d^{4}\right) .
$$

From the above expansion we see that equation (8) behaves like $\sim \beta d$ as $d \rightarrow 0$. However, because $\beta$ is of a very small value, the $d^{2}$ term is important even in the $d \rightarrow 0$ limit. The total energy approaches to the the single atom energy $\varepsilon_{0}^{(N)}$ more likely as a parabolic function.

In conclusion, we calculated the ground-state energy of a classical artificial molecule based on the analytical investigation and the MD simulation. For the small molecules, we obtained analytical expressions for the exact groundstate energy as a function of inter-atom distance. By fitting the MD data for molecules of $N=4$ to 128 , we were able to find out a simple formula (Eqs. (8-10)) to approximate the ground-state energy of the classical molecules for any values of the particle number $N$ and inter-atom distance $d$. This function is reliable for the particle systems obeying classical mechanics since it fits very well the MD data within an error less than $2 \%$. We expect that our results would be interesting for those studies on artificial molecules constituted by coupled clusters of ions or colloidal particles in traps or by coupled quantum dots in semiconductor structures in high magnetic field regime.

This work was supported by FUNAPE/UFG, FAPESP, CAPES and CNPq, Brazil.

\section{References}

1. R. Bubeck, C. Bechinger, S. Neser, P. Leiderer, Phys. Rev. Lett. 82, 3364 (1999); Q.-H. Wei, C. Bechinger, D. Rudhardt, P. Leiderer, Phys. Rev. Lett. 81, 2606 (1998)

2. B.G. Levi, Phys. Today 41, 17 (1988)

3. W.-T. Juan, Z.-H. Huang, J.-W. Hsu, Y.-J. Lai, I. Lin, Phys. Rev. E 58, R6947 (1998)

4. S.J. Gilbert, J.J. Bolinger, D.J. Wineland, Phys. Rev. Lett. 60, 2022 (1988); W.M. Itano, J.J. Bollinger, J.N. Tan, B. Jalenkovic, D.J. Wineland, Science 297, 686 (1988); P. Ludwig, S. Kosse, M. Bonitz, Phys. Rev. E 71, 046403 
(2005); B.G.A. Brito, J.N.T. Rabelo, L. Cândido, Phys. Lett. A 353, 70 (2006)

5. Y. Kondo, J.S. Korhonen, M. Krusius, V.V. Dimitriev, E.V. Thuneberg, G.E. Volovik, Phys. Rev. Lett. 68, 3331 (1992)

6. P. Leiderer, W. Ebner, V.B. Shikin, Surf. Sci. 113, 105 (1987)

7. M.A. Reed, W.P. Kirk, Nanostructure Physics and Fabrication (Academic Press, Boston, 1989)

8. R. Ashoori, Nature 379, 413 (1996)

9. M. Marlo, M. Alatalo, A. Harju, R.M. Nieminen, Phys. Rev. B 66, 155322 (2002)

10. D.G. Austing, T. Honda, K. Muraki, Y. Tokura, S. Tarucha, Physica B 249, 206 (1998)

11. V.M. Bedanov, F.M. Peeters, Phys. Rev. B 49, 2667 (1994); F. Bolton, U. Rossler, Superlatt. Microstruct. 13, $139(1993)$
12. L. Cândido, J.P. Rino, N. Studart, F.M. Peeters, J. Phys.: Condens. Matter 10, 11627 (1998)

13. J.J. Thomson, Philos. Mag. 39, 237 (1904)

14. B. Partoens, V.A. Schweigert, F.M. Peeters, Phys. Rev. Lett. 79, 3990 (1997)

15. W.P. Ferreira, G.A. Farias, H.A. Carmona, F.M. Peeters, Solid Stat. Commun. 122, 665 (2002)

16. W.P. Ferreira, A. Matulis, G.A. Farias, F.M. Peeters, Phys. Rev. E 67, 046601 (2003)

17. C. Yannouleas, U. Landman, Phys. Rev. 66, 115315 (2002)

18. J.P. Perdew, Y. Wang, Phys. Rev. B 45, 13244 (1992); J.P. Perdew, J.A. Chevary, S.H. Vosko, K.A. Jackson, M.R. Pederson, D.J. Singh, C. Fiolhais, Phys. Rev. B 46, 6671 (1992); C.C. Lee, W. Yang, R.G. Parr, Phys. Rev. B 37, 785 (1988)

19. J.D. Jackson, Classical Electrodynamics (John Wiley, 1975), Chap. 4, p. 136 